\title{
Auto-atribución de comportamientos socialmente responsables de estudiantes de carreras del área de ciencias sociales $^{1}$
}

\author{
María José Bustamante Oliveros* \\ Gracia Navarro Saldaña**
}

\begin{abstract}
RESUMEN
En el presente artículo se presentan los resultados de un estudio que describe la autoatribución de comportamientos socialmente responsables e intenciones a la base y su relación con factores biológicos, ambientales y cognitivos, en una muestra de 261 estudiantes de la Facultad de Ciencias Sociales de la Universidad de Concepción. Para la recolección de datos se utilizó el Cuestionario de Autoatribución de Comportamientos Socialmente Responsables y una Encuesta de antecedentes personales.
\end{abstract}

Palabras clave: Responsabilidad Social / Competencias Profesionales Generales / Formación Universitaria / Capital Humano Avanzado

\section{ABSTRACT}

The present study describes the self-attribution of socially responsible behaviors and intentions and their relation with biological, environmental and cognitive factors, in a sample of 261 students of the Faculty of Social Sciences of the University of Conception. For data collection, the Questionnaire of Self -attribution of Socially Responsible Behaviors and a Survey for personal records were used.

Key words: Social Responsibility / General Professional Competencies / University Training / Advanced Human Capital.

\footnotetext{
Este artículo fue presentado originalmente con el nombre de: "Auto-atribución de comportamientos socialmente responsables y su relación con factores biológicos, ambientales y cognitivos en alumnos de la facultad de ciencias sociales - Universidad de Concepción"

- Chilena. Trabajadora Social. Coordinadora de Proyecto-Corporación SEDE] Concepción. E-mail: mariajosebusta@gmail.com

* Chilena. Psicóloga. Profesora Asociada Departamento de Psicología UEDC. Directora Programa de estudios sobre la Responsabilidad Social Universidad de Concepción. E-mail: gnavarro@udec.cl
} 


\section{Introducción}

I as universidades, dedicadas a generar conocimiento y a formar profesionales, han incorporado recientemente, en sus ejes y programas de estudios, temáticas en torno a la formación profesional integral, involucrando valores como la equidad, tolerancia y solidaridad; valores enmarcados en el concepto de Responsabilidad Social (RS), que es identificada en la formación académica como competencia genérica indispensable a desarrollar en los futuros profesionales, respondiendo a los desafíos y nuevas exigencias educacionales del país que buscan el establecimiento de mínimos formativos favorables a la construcción de una sociedad más justa. A su vez, en el desarrollo de la Responsabilidad Social, no solo influirían la educación formal entregada, puesto que los factores biológicos, ambientales y cognitivos del estudiante podrían determinar el nivel de desarrollo que alcance en dicha competencia.

En base a lo anterior, con el fin de describir la Auto-atribución de Frecuencia e Intención de Comportamientos Socialmente Responsables, y su relación con algunas variables cognitivas, ambientales y biológicas, se realizó un estudio en 261 estudiantes de la Facultad de Ciencias Sociales de la Universidad de Concepción (UdeC).

Los resultados obtenidos y aquí presentados pueden ser considerados orientaciones, tanto para la Facultad de Ciencias Sociales de la Universidad de Concepción como para las universidades que se encuentren en la tarea de realizar cambios programáticos relacionados con la formación valórica y para los docentes que se han adscrito al desafío de la formación profesional, en concordancia con la Declaración Mundial sobre Educación Superior para el siglo XXI, aprobada en la Conferencia Mundial sobre Educación Superior organizada por UNESCO.

Los resultados permiten tener un diagnóstico inicial y final de los estudiantes, en base al cual tomar decisiones educacionales en el tema; también permiten identificar variables que, por su relación con la autoatribución de comportamientos socialmente responsables e intenciones a la base, serían necesarias de considerar a la hora de trabajar en la formación de competencias en RS. 


\section{Capital humano y responsabilidad social}

La Educación es la base de la Teoría del Capital Humano entendida como mecanismo de formación que permite el crecimiento del país con desarrollo económico, justicia y equidad. Se considera un componente de desarrollo humano y elemento fundamental del desarrollo social sostenible.

Actualmente, se manifiestan con gran fuerza iniciativas generadoras de inversión en Capital Humano en la Educación, denominado "Capital Humano Avanzado" basado en la necesidad de generar capital humano, favorable al crecimiento social del país. En Chile, cálculos recientes revelan que cada año de educación básica otorga 4\% extra de renta al país; la enseñanza media redunda en 10\% más de ingresos, como también cada año en la universidad genera $20 \%$ más en la remuneración (Brunner, 2003).

En este contexto, a través de la educación formal y de la experiencia vital de cada uno, Chile debiera poder contar con personas desarrolladas, capaces de contribuir al desarrollo de otros y desempeñarse efectivamente en una situación laboral. Las universidades enfrentan, entonces, la búsqueda de calidad y formación que responda a un modelo de enseñanza centrado en el aprendizaje, para lo que se han considerado múltiples cambios en los procesos formativos curriculares basados en competencias genéricas y específicas.

El concepto de competencia establecido por la Universidad de Concepción, a través de la Dirección de Docencia², es mencionado dentro de las Políticas de Docencia de Pregrado, identificada como el "conjunto de conocimientos, procedimientos y actitudes combinados, coordinados e integrados en la acción, adquiridos a través de la experiencia (formativa y no formativa) que permite al individuo resolver problemas específicos de forma autónoma y flexible en contextos singulares"3.

\footnotetext{
Organismo dependiente de Vicerrectoría, responsable de coordinar la docencia de pregrado de la Udec, tiene dentro de sus funciones estudiar las políticas de desarrollo de docencia de pregrado, los currículos de las carreras de pregrado, generar estudios tendientes a evaluar las actividades relativas a este tipo de docencia, entre otras funciones. Información Obtenida en Pagina WEBhttp://www2. udec.cl/ dirdoc/docencia.php, en el mes de abril de 2007.

3 Universidad de Concepción. Dirección de Docencia, definición extraída de ponencia "Política de Docencia de Pregrado, del Director de Docencia Prof. José Sánchez Henríquez, en marzo de 2007". Disponible En: http://www2.udec.cl/ dirdoc/ perfeccionamiento/seminarios.php.
} 
Las competencias genéricas podrían ser consideradas como "batería" de habilidades personales que no sólo se relacionan con el área de formación universitaria, permitiendo a un individuo desenvolverse efectiva e integralmente ante situaciones no solo profesionales. Quienes cuenten con competencias genéricas disponen de habilidades, destrezas y actitudes necesarias para ejercer una profesión, resolver problemas de forma autónoma-flexible, como también estarían capacitados para colaborar en su entorno profesional y en la organización del trabajo; entre dichas competencias se distingue a la responsabilidad social.

Existen numerosos acercamientos teóricos para el concepto de Responsabilidad Social o RS. Navarro (2003; 2006) la define como la capacidad y obligación de cada persona, de responder ante la sociedad por acciones $u$ omisiones que se traducen en un compromiso personal con los demás y se refleja en comportamientos morales que orientan las actividades individuales y colectivas en un sentido, que permitan a todos igualdad de oportunidades para desarrollar sus capacidades y satisfacer sus necesidades. Incluye actitudes, valores y comportamientos concretos a través de los cuales se ejerce.

La RS se desarrolla y sería influenciada por factores biológicos, cognitivos y ambientales, como la educación, las interacciones personales y la experiencia vital, requiriendo del logro de tareas o ajustes conducentes a la llamada autonomía socialmente responsable, esto es "la capacidad, deber y derecho a dirigir la propia vida, a partir de la toma de decisiones que consideren y evalúen la situación, el contexto, el propio plan de vida; la evaluación de las consecuencias de estas decisiones, no sólo en sí mismo, sino también en los demás, en el plan de vida y bienestar de los otros, haciéndose cargo de estas consecuencias" (Navarro, 2003: 28).

Un mismo comportamiento puede ser o no ser socialmente responsable, lo que va a depender de las intenciones que tenga a la base la persona que lo ejecuta. Así, un comportamiento puede tener la intención de beneficio propio (individualismo), beneficio para los demás o beneficio común (beneficia los demás y también a mí). El comportamiento socialmente responsable es aquel que tiene a la base la intención de beneficio común (Navarro, 2006).

Por otra parte, en la medida en que las personas intercambian experiencias con otros, desarrollan el sentido de compartir, interna- 
lizando aspectos morales sobre normas de cooperación y respeto mutuo, donde, junto con enfrentar la toma de decisiones durante su desarrollo, surge la preocupación por otros, exigiendo un compromiso y determinación de objetivos personales que se encuentren en la misma dirección de los objetivos de la sociedad.

Según Navarro (2003; a), la Responsabilidad Social es un valor que se manifiesta en cada persona adulta cuando logra tomar conciencia de que tiene el deber y la capacidad de responder ante otros, y para ser considerada como una persona socialmente responsable, no solo requiere conocer el concepto; debe ejercer conductas socialmente responsables.

\section{Educación para la Responsabilidad Social}

La Educación permite el desarrollo de valores y competencias que favorecen la integración de las personas a la sociedad. Así, la responsabilidad social se vuelve un concepto fundamental que surge primeramente en la familia como agente de cambio social y de transmisión de valores, como también surge en los procesos formativos de educación formal.

La educación en responsabilidad social se plantea en términos de proximidad y compromiso personal con otros, siendo ello el motor predominante en las personas que se interesan por hacer de su vocación su profesión, cobrando relevancia la educación integral y la educabilidad de las emociones.

La RS se ha vuelto un desafío para las instituciones educativas superiores, que tienen a la base de su tarea entregar-fortalecer valores, supuestos y prácticas, que han sido evaluados y replanteados, a fin de favorecer el desarrollo de la comunidad educativa y de la sociedad donde se encuentran insertas, viéndose en la necesidad de revisar los currículos (mallas) de formación incorporando conceptos de desempeño y ejercicio de la Responsabilidad, para alcanzar la formación de profesionales y técnicos capaces de responder a las necesidades sociales actuales, a través del desarrollo de habilidades a nivel cognitivo, afectivo y conductuales, que indudablemente requiere cambios en las estrategias de enseñanza, evaluación y en la relación profesor-alumno (Navarro, 2003). 


\section{Factores asociados a la Responsabilidad Social}

Factores Biológicos: Según Barudy (2005), el desarrollo y diferencia de roles entre hombres y mujeres estarían fuertemente determinados por factores biológicos que influyen en la vida social de ellas y en su descendencia. Algunos estudios señalan que las mujeres, especialmente quienes han sido madres, priorizan el cuidado y el buen trato de sus hijos, lo que se explicaría por la capacidad del organismo femenino de producir la hormona Oxitonita, que predispone a las personas a vincularse en relaciones de ayuda y asociación, permitiendo mayor número de comportamientos socialmente responsables al poseer capacidad natural de regular su conducta en función de terceros o conductas sociales altruistas.

Factores Ambientales: Podría esperarse que varios factores ambientales influyan en la responsabilidad social de los estudiantes. Entre ellos, el estudio busca contrastar la existencia de relaciones significativas con los siguientes.

- El Lugar de Residencia de los alumnos previo a su incorporación a la vida universitaria: la "Ciudad de Origen" considerada factor ambiental territorial, que proporciona experiencias e interacciones culturalmente variadas.

- El "Apoyo Económico Universitario" entregado por la UdeC a sus alumnos para cursar la educación superior: en la medida que éste existe, favorecería el desarrollo de actividades académicas, propiciando un ambiente facilitador de la formación al cubrir parte de una recurrente necesidad universitaria (recurso económico). La inversión en educación y en personas con aptitudes, se liga a la ejecución de la responsabilidad social al generar espacios, aprovechar y no desperdiciar las capacidades cognitivas, intelectuales y talentos de los ciudadanos, especialmente de los jóvenes.

- La pertenencia a una familia nuclear con ejercicio de voluntariado: la familia se reconoce socialmente como el principal espacio educador y transmisor de valores; por tanto, se esperaría que la familia que realiza acciones de voluntariado estaría mostrando modelos de compromiso social y transmitiendo mensajes positivos relacionados con el ejercicio de la responsabilidad social que tendrían influencia en el joven. 
- Tipo de Enseñanza Media de procedencia (particular, público o particular-subvencionado). Según Bronfenbrenner y Morris (1998, en Navarro, 2003) al logro de la conducta socialmente responsable contribuyen todos los agentes de socialización, familia, escuela, pares, medios de comunicación, entre otros. Si existen diferencias significativas en el logro de objetivos académicos en la educación municipal, particular pagada y particular subvencionada, demostradas año tras año a través de los resultados del SIMCE, sería esperable que en Chile también existan diferencias en el logro de objetivos transversales, entre los cuales se puede mencionar el compromiso con los demás o la responsabilidad social.

Factores Cognitivos: La formación que entrega cada carrera universitaria influye en la manera de conocer, entender e interpretar el mundo, influyendo en el comportamiento de sus estudiantes. Por consiguiente, podría estar relacionada con la autoatribución de comportamiento socialmente responsable e intenciones a la base. Con ello, podría esperarse un efecto diferencial de acuerdo al tiempo que el alumno lleve estudiando la carrera, al considerar la exposición a una determinada cultura organizacional, a determinados programas de estudio o modelo educativo, a determinadas interacciones interpersonales y diferentes oportunidades para ejercer la RS (alumno de primer o cuarto año), junto a los logros alcanzados en su formación, los que en esta investigación se expresan a través del rendimiento académico (notas).

Con respecto a la formación profesional, la UdeC declara como misión la formación de "mujeres y hombres altamente calificados, íntegros e integrales, comprometidos con valores que defienden y difunden activamente; que ven su profesión como una posibilidad de servicio a los demás; capaces de aportar como ciudadanos a la construcción de la sociedad y de responder creativamente a los desafíos de un proyecto país"(UdeC, 2006: 1), siendo este un elemento gravitante en la visión de una universidad socialmente responsable, que debiera impregnar la formación en todas sus carreras, se esperaría encontrar algunas diferencias entre carreras.

Por otra parte, considerando y articulando los conceptos planteados, el objetivo de esta investigación es "Describir la autoatribución de comportamientos socialmente responsables e intenciones a la base y su relación con factores biológicos, ambientales y cognitivos 
en alumnos de la Facultad de Ciencias Sociales de la Universidad de Concepción". Para alcanzar este objetivo se buscó determinar la autoatribución de comportamientos socialmente responsables e intenciones a la base en estudiantes de la facultad de Ciencias Sociales de la UdeC. Como asimismo explorar si existen diferencias significativas en la autoatribución de comportamientos socialmente responsables e intenciones a la base según la carrera. Finalmente se busca establecer si existe relación entre la autoatribución de comportamientos socialmente responsables e intenciones a la base y el sexo del alumno, el lugar de residencia previo, el apoyo económico recibido de la Universidad, su pertenencia a una familia con ejercicio de voluntariado, el ejercicio de voluntariado del alumno, el tipo de enseñanza media de procedencia, el nivel o curso actual y su rendimiento académico (notas).

\section{Metodología empleada}

Para desarrollar este estudio se conformó una muestra a partir de los siguientes criterios:

- Alumnos de las carreras de la Facultad de Ciencias SocialesUdeC.

- Alumnos matriculados el segundo semestre académico de 2005, en $1^{\circ}$ ó $4^{\circ}$ año en las carreras de Psicología, Periodismo, Servicio Social, Sociología y Antropología.

- Alumnos que, cursando el segundo semestre de 2005, asisten regular y espontáneamente a las asignaturas inscritas.

\begin{tabular}{|l|c|c|c|}
\hline Carrera & ler año & 4to año & Total \\
\hline Ser. Social & 38 & 38 & 76 \\
\hline Psicología & 46 & 26 & 72 \\
\hline Sociología & 27 & 12 & 39 \\
\hline Periodismo & 32 & 20 & 52 \\
\hline Antropología & 22 & - & 22 \\
\hline Total & 165 & 96 & 261 \\
\hline
\end{tabular}

Finalmente, la muestra quedó constituida por 261 alumnos, 57\% mujeres y $43 \%$ hombres, tamaño que se configuró al momento de la aplicación del instrumento, al cuidar la característica de estudio natural. 


\section{Variables en Estudio}

Entre las variables estudiadas, se consideró como variables dependientes:

- Autoatribución de Frecuencia de comportamiento socialmente responsable.

- Autoatribución de Intención a la base del comportamiento socialmente responsable.

Mientras que las variables Independientes fueron clasificadas en factores:

- Factores Cognitivos: Carrera. Año-nivel Universitario. Rendimiento académico.

- Factores Ambientales: Apoyo económico universitario. Familia Nuclear con ejercicio de voluntariado. Participación del Alumno en actividades de Voluntariado. Tipo de Enseñanza Media de procedencia Ciudad de origen.

- Factores Biológicos: Sexo.

\section{Instrumentos}

Para la recolección de datos, se utilizó el Cuestionario de Autoatribución de Comportamientos Socialmente Responsables y una encuesta breve de Antecedentes personales de los alumnos.

Instrumento 1: Cuestionario de Autoatribución de Comportamientos Socialmente Responsables.

El Instrumento se compone de dos escalas, midiendo Auto-atribución de Frecuencia (Escala 1) e Intención (Escala 2) de RS; es una escala con formato Likert construido en el año 2003 que ha mostrado ser altamente recomendable para otros estudios de responsabilidad social ${ }^{4}$. Ambas escalas están formadas por 40 ítemes, distribuidos en 10 subescalas (Responsabilidad Laboral, Actividades de Voluntariado, Ayuda Social, Actividades Religiosas, Convivencia Social, Responsabilidad Cívica, Autocuidado, Desarrollo Cultural, Ecología y Medio Ambiente y Respeto por Espacios Compartidos), arrojando un puntaje global por escala y uno por subescala.

Alpha de Cronbach escala 1: 0.82 -escala 2: 0.76; Coeficiente de división por mitades escala 1: 0.86 - escala 2: 0.72; Confiabilidad interna escala 1: 0.75 escala 2: 0.74 ; relación entre ambas escalas $r$ Square $=0,2554<0.0044$ 
Los resultados son analizados según la variabilidad de los puntajes de cada escala; Escala 1 de Frecuencia entre 1-5, y la Escala 2 Intención entre 1-4.

Escala 1 Frecuencia:

\begin{tabular}{|c|c|c|c|c|c|c|}
\hline $\begin{array}{c}\text { Menor Frecuencia } \\
\text { de Autoatribución } \\
\text { CSR }\end{array}$ & Nunca & $\begin{array}{c}\text { Casi } \\
\text { nunca }\end{array}$ & A veces & $\begin{array}{c}\text { Casi } \\
\text { Siempre }\end{array}$ & Siempre & $\begin{array}{c}\text { Mayor Frecuencia } \\
\text { de Autoatribución } \\
\text { CSR }\end{array}$ \\
\hline & 1 & 2 & 3 & 4 & 5 & \\
\hline
\end{tabular}

Escala 2 Intención:

Esta escala evalúa las intenciones de los comportamientos identificados según frecuencia en la escala.

\begin{tabular}{|c|c|c|c|c|c|}
\hline $\begin{array}{c}\text { Menor Intención } \\
\text { de Autoatribución } \\
\text { CSR }\end{array}$ & $\begin{array}{c}\text { Beneficio } \\
\text { Personal }\end{array}$ & $\begin{array}{c}\text { No tengo clara la } \\
\text { intención }\end{array}$ & $\begin{array}{c}\text { Beneficio } \\
\text { para otros }\end{array}$ & $\begin{array}{c}\text { Beneficio } \\
\text { Mutuo }\end{array}$ & $\begin{array}{c}\text { Mayor Intención } \\
\text { de Autoatribución }\end{array}$ \\
\hline
\end{tabular}

Instrumento 2: Encuesta de antecedentes personales de los estudiantes.

Para recoger la información respecto a las variables independientes identificadas, se creó una encuesta simple sobre los antecedentes personales relevantes de cada alumno, que fue adjuntada al instrumento anterior permitiendo un solo momento de recolección de datos. Las variables independientes se distinguen en 9 ítemes (Carrera, Sexo, Años de permanencia en la universidad, Apoyo económico universitario (Beca-Crédito), Rendimiento Académico, Familia Nuclear con ejercicio de voluntariado, Alumno involucrado en actividades de voluntariado, Tipo de educación media de Procedencia y Ciudad de origen)

\section{Procedimiento}

Para acceder a la recolección de información, se envió una carta a cada Director-Jefe de Carrera de la Facultad de Ciencias Sociales, solicitando su colaboración y autorización para realizar la aplicación de los instrumentos durante el desarrollo de una asignatura obligatoria o de alta asistencia, quienes autorizaron la aplicación. Al momento de la recolección de datos, se informó primero al docente de la asignatura sobre los objetivos de la investigación, quienes brindaron el tiempo suficiente para la aplicación, procedimiento que bordeó los 45 minutos por curso. 
La característica de aplicación masiva requirió de solo un aplicador del instrumento, que concretó 9 aplicaciones durante los días 4 y 23 de noviembre de 2005. Los alumnos contestaron por sí mismos el instrumento. Posteriormente se dio inicio al Pre-Proceso de la información consistente en: Construir Códigos, Codificar y Digitar la Codificación de los resultados. Para finalmente realizar el Proceso computacional y consiguiente análisis estadístico.

\section{Procesamiento de los Datos}

El proceso de análisis de la información se realizó a través del Software Estadístico "Statistical Analysis System" -SAS- que proporciona cálculo de frecuencia y porcentaje. Para realizar comparación, se utilizó Análisis de Covarianza puesto que la variable dependiente utilizada autoatribución de comportamiento socialmente responsable se compone de dos escalas globales (Frecuencia e Intención), cada una de ellas compuestas por 10 sub-escalas, más la escala total, obteniendo 11 variables dependientes en ambas escalas.

Luego se estableció la correlación entre las variables independientes definidas, entregando antecedentes de cálculo existente entre variables continuas, a fin de conocer su influencia o no en la autoatribución del CSR.

Para obtener antecedentes medidos en frecuencia relacionadas a las variables independientes categóricas: carrera, apoyo económico universitario, tipo de Enseñanza media de procedencia; se empleó análisis de varianza ANOVA. Así también, para medir intención, se utilizó el mismo análisis para las variables apoyo económico universitario y tipo de enseñanza media de procedencia.

Para obtener información relativa a comparación de la frecuencia e intención de autoatribución con las variables independientes: sexo, nivel-año universitario, rendimiento académico, familia nuclear con actividades de voluntariado, actividad de voluntariado del alumno y ciudad de origen, se empleó Análisis de T-student.

\section{Resultados obtenidos}

La Responsabilidad Social se desarrolla y es el resultado de actos individuales y grupales realizados a partir de la toma de decisiones tendientes al beneficio común, incluyendo a los demás y a quien(es) decide(n) ejecutar estos actos. Ello implica asumir la responsabili- 
dad con las consecuencias de acciones u omisiones, lo que es posible de alcanzar solo al término de la edad juvenil, etapa en la que se encuentran los universitarios. Los resultados obtenidos son orientados dando respuesta a los objetivos específicos establecidos.

\section{Autoatribución de comportamientos socialmente responsables e intenciones a la base en estudiantes de la Facultad de Ciencias Sociales de la UdeC}

En la Escala 1 (ver Tabla 1), se presentó una media general de 3,45 puntos en una gama posible de variación ${ }^{5}$ de 1 a 5 ; mientras que en la Escala 2 (ver Tabla 2), los sujetos presentaron una media general de 2,38 puntos de autoatribución de intenciones, la variabilidad en esta escala por ítem es 1 a 4 puntos, partiendo de intenciones más individualistas a más colectivistas; siendo las intenciones de la escala 2 el trasfondo de los comportamientos de la escala 1. Ambas Escalas ubican el resultado global por sobre la media posible de alcanzar.

Tabla 1. Escala 1: Frecuencia de Autoatribución de Comportamientos Socialmente Responsable

\begin{tabular}{|l|c|c|c|c|}
\hline Sub- escalas & N & $\begin{array}{c}\text { Rango } \\
\text { Mínimo }\end{array}$ & $\begin{array}{c}\text { Rango } \\
\text { Máximo }\end{array}$ & Media \\
\hline Responsabilidad Académica & 261 & 8 & 20 & 4.14 \\
\hline Actividad de voluntariado & 260 & 3 & 20 & 2.36 \\
\hline Ayuda Social & 261 & 4 & 18 & 2.55 \\
\hline Actividades Religiosas & 254 & 1 & 20 & 2.20 \\
\hline Convivencia Social & 261 & 9 & 20 & 4.29 \\
\hline Responsabilidad Cívica & 261 & 5 & 20 & 3.48 \\
\hline Auto Cuidado & 261 & 8 & 20 & 4.22 \\
\hline Desarrollo Cultural & 261 & 7 & 20 & 3.67 \\
\hline Ecología y Medio Ambiente & 261 & 8 & 20 & 3.33 \\
\hline Respeto por los espacios compartidos & 261 & 5 & 20 & 4.33 \\
\hline Escala 1. Promedio & 260 & 5.8 & 19.8 & 3.45 \\
\hline
\end{tabular}

En autoatribución destacan, como puntajes extremos, la sub-escala Actividades Religiosas como la de menor autoatribución 2.20, en

\footnotetext{
La variación también puede ser considerando entre los rangos 50 y 200 como mínimo y máximo respectivamente, los que surge de la sumatoria de los resultados de cada ítem (40) donde a mayor puntaje existe mayor frecuencia autoatribuida; en tanto la escala 2, alcanza rangosa totales entre 40 y 160, en la sumatoria de los ítem, partiendo de intención individualista a más colectivista.
} 
oposición a la sub-escala "Respeto por los Espacios Compartidos" que alcanza el mayor puntaje 4.33.

En Intención se encuentran la sub-escala "responsabilidad académica" como la sub-escala con actos de connotación mayormente individualistas, en oposición a la sub-escala "autocuidado" que refleja mayormente la intención de ser actos colectivistas (Beneficio Mutuo).

Tabla 2. Escala 2: Intención de Autoatribución de Comportamientos Socialmente Responsables

\begin{tabular}{|l|c|c|c|c|}
\hline Sub- escalas & N & $\begin{array}{c}\text { Rango } \\
\text { Mínimo }\end{array}$ & $\begin{array}{c}\text { Rango } \\
\text { Máximo }\end{array}$ & Media \\
\hline Responsabilidad Académica & 261 & 2 & 16 & 1.23 \\
\hline Actividad de voluntariado & 248 & 1 & 16 & 2.13 \\
\hline Ayuda Social & 256 & 2 & 16 & 2.85 \\
\hline Actividades Religiosas & 229 & 1 & 15 & 2.46 \\
\hline Convivencia Social & 261 & 4 & 16 & 2.25 \\
\hline Responsabilidad Cívica & 260 & 1 & 15 & 2.41 \\
\hline Auto Cuidado & 260 & 2 & 16 & 3.13 \\
\hline Desarrollo Cultural & 260 & 2 & 14 & 2.56 \\
\hline Ecología y Medio Ambiente & 261 & 3 & 16 & 2.26 \\
\hline Respeto por los espacios compartidos & 261 & 4 & 16 & 2.28 \\
\hline Escala 2 & 255.7 & 2.2 & 15.6 & 2.38 \\
\hline
\end{tabular}

\section{Autoatribución de comportamientos socialmente responsables e intenciones a la base según la carrera}

En la categoría Carrera relacionada con la escala 1 muestra dos grupos, los que mantienen diferencia significativa de $\operatorname{Pr}>0.0010$ en autoatribución de comportamientos socialmente responsables.

El primer grupo está compuesto por las carreras de Servicio Social, Antropología, Psicología y Periodismo, donde Servicio Social presenta la media más alta, liderando el grupo A con 141.7 puntos (los puntajes entregados se presentan con relación a los puntajes máximos obtenidos en la sumatoria de todos los ítem 40 a 200). Diferenciándose significativamente del grupo B, conformado solo por la carrera de Sociología. 
Tabla N³: Análisis de Varianza (ANOVA) de Frecuencia de Auto-atribución de comportamiento socialmente responsable de los alumnos de la Facultad de CS. Sociales de la UDEC; por Carrera; 2005.

\begin{tabular}{|l|l|c|c|c|c|c|c|c|}
\hline \multicolumn{2}{|l|}{ CARRERA } & \multirow{2}{*}{$\mathrm{N}$} & $\%$ & \multicolumn{5}{|c|}{ FRECUENCIA } \\
\cline { 5 - 6 } & & & Media & Duncan & F Value & Pr $>$ F & Var \\
1. & Servicio Social & 76 & 29 & 141.8 & A & 4.79 & 0.0010 & VS \\
\hline 2. & Antropología & 22 & 28 & 137.5 & & & & \\
\hline 3. & Psicología & 71 & 15 & 136.4 & & & & \\
\hline 4. & Periodismo & 52 & 20 & 136.3 & & & & \\
\hline 5 & Sociología & 39 & 08 & 129.5 & B & & & \\
\hline
\end{tabular}

\section{Autoatribución de comportamientos socialmente responsables e intenciones a la base según variables independientes}

- Relación entre sexo del alumno y autoatribución de comportamientos socialmente responsables e intenciones a la base

Al comparar los puntajes de media en la Escala 1, entre personas de sexo femenino y masculino, el que es considerado en el estudio Factor Biológico, se observa diferencia significativa a favor de las mujeres quienes presentan media de 142 puntos, indicando que se auto-atribuyen con mayor frecuencia comportamientos socialmente responsables en comparación con los hombres. Así lo muestra la ponderación mediante la prueba t 0.0001. En la Escala de Intención, tanto hombres como mujeres no presentan diferencia significativa.

Tabla N 4 : T- Student de Frecuencia de auta-atribución e Intención socialmente responsable de los alumnos de la Facultad de CS. Sociales de la UDEC; por Sexo; 2005.

\begin{tabular}{|c|c|c|c|c|c|c|c|c|c|}
\hline \multirow{2}{*}{\multicolumn{2}{|c|}{ SEXO }} & \multirow[b]{2}{*}{$\mathrm{N}$} & \multirow[b]{2}{*}{$\%$} & \multicolumn{6}{|c|}{ FRECUENCIA (escalal) } \\
\hline & & & & Media & Std dev & $\operatorname{Pr}>\mathrm{F}$ & Var & $t$ & $\operatorname{Pr}>t$ \\
\hline 1. & Femenino & 149 & 57 & 141.7 & 12.5 & 0.0163 & VS & 6.06 & 0.0001 \\
\hline 2. & Masculino & 112 & 43 & 130.9 & 15.5 & & & & \\
\hline
\end{tabular}

- Relación entre pertenencia a una familia con ejercicio de voluntariado y autoatribución de comportamientos socialmente responsables e intenciones a la base

Se encontró diferencia significativa en la variable "Pertenencia del alumno a familia nuclear con ejercicio de voluntariado" considerada factor ambiental, puesto que la Escalal alcanza en la prueba T 0.0025 demostrando influencia de esta variable en la auto-atribución 
de comportamiento socialmente responsable, alcanzado una media de 141 puntos, en frecuencia, contrastando con los 135 puntos para aquellos con familias sin desarrollo de voluntariado. Respecto de la escala de intención, no se observan relación significativa.

Tabla N5: T- Student de Frecuencia e Intención de autoatribución de comportamiento socialmente responsable de los alumnos de la Facultad de CS. Sociales de la UDEC; por Familia Nuclear con Ejercicio de Voluntariado; 2005.

\begin{tabular}{|c|c|c|c|c|c|c|c|c|c|}
\hline \multirow{2}{*}{\multicolumn{2}{|c|}{$\begin{array}{l}\text { FAMILIA CON PARTICIPACIÓN } \\
\text { EN ACTIVIDADES DE } \\
\text { VOLUNTARIADO }\end{array}$}} & \multirow[b]{2}{*}{$\mathrm{N}$} & \multirow[b]{2}{*}{$\%$} & \multicolumn{6}{|c|}{ FRECUENCIA } \\
\hline & & & & Media & Std dev & \multirow{2}{*}{$\begin{array}{l}\operatorname{Pr}>F \\
0.6253\end{array}$} & \multirow{2}{*}{$\begin{array}{l}\text { Var } \\
\text { VS }\end{array}$} & \multirow{2}{*}{$\begin{array}{c}t \\
3.06\end{array}$} & \multirow{2}{*}{$\begin{array}{c}\text { Pr }>t \\
0.0025\end{array}$} \\
\hline 1. & SI & 78 & 30 & 141.3 & 15.123 & & & & \\
\hline 2. & NO & 178 & 70 & 135.2 & 14.463 & & & & \\
\hline
\end{tabular}

- Relación entre ejercicio de voluntariado del alumno y autoatribución de comportamientos socialmente responsables e intenciones a la base

Con respecto a los alumnos que declaran participar en actividades de voluntariado, factor ambiental, se identifica una diferencia superior de 11.2 puntos en Frecuencia de autoatribución y 4.7 en Intención por sobre quienes no las realizan, existiendo varianza significativa de 0.0001 en Frecuencia, y en Intención que alcanza 0.0105 en la prueba de varianza.

Tabla $N^{\circ}$ 6: T- Student de Frecuencia e Intención de autoatribución de comportamiento socialmente responsable de los alumnos de la Facultad de CS. Sociales de la UDEC; por Ejercicio de Voluntariado de Alumnos; 2005.

\begin{tabular}{|c|c|c|c|c|c|c|c|c|c|c|c|c|c|c|c|}
\hline \multirow{2}{*}{\multicolumn{2}{|c|}{$\begin{array}{l}\text { ALUMNOS CON } \\
\text { PARTICIPACIÓN EN } \\
\text { ACTIVIDADES DE } \\
\text { VOLUNTARIADO }\end{array}$}} & \multirow[b]{2}{*}{$\mathrm{N}$} & \multirow[b]{2}{*}{$\%$} & \multicolumn{6}{|c|}{ FRECUENCIA } & \multicolumn{6}{|c|}{ INTENCIÓN } \\
\hline & & & & Media & $\begin{array}{l}\text { Std } \\
\text { dev }\end{array}$ & \multirow[t]{2}{*}{$\begin{array}{l}\mathrm{Pr}>\mathrm{F} \\
0.0638\end{array}$} & \multirow[t]{2}{*}{$\begin{array}{l}\text { Var } \\
\text { VS }\end{array}$} & \multirow[t]{2}{*}{$\begin{array}{c}\mathrm{t} \\
6.29\end{array}$} & \multirow[t]{2}{*}{$\begin{array}{c}\operatorname{Pr}>t \\
0.0001\end{array}$} & Media & $\begin{array}{l}\text { Std } \\
\text { dev }\end{array}$ & \multirow[t]{2}{*}{$\begin{array}{l}\operatorname{Pr}>\mathrm{F} \\
0.0248\end{array}$} & \multirow[t]{2}{*}{$\begin{array}{l}\text { Var } \\
\text { VS }\end{array}$} & \multirow[t]{2}{*}{$\mid \begin{array}{c}\mathrm{T} \\
2.58\end{array}$} & \multirow[t]{2}{*}{$\begin{array}{c}\text { Pr>lt } \\
0.0105\end{array}$} \\
\hline 1. & SI & 101 & 39 & 143.9 & 12.4 & & & & & \begin{tabular}{|l|}
90.45 \\
\end{tabular} & 13.08 & & & & \\
\hline & NO & 158 & 61 & 132.7 & 14.8 & & & & & 85.72 & 16.11 & & & & \\
\hline
\end{tabular}

- Relación entre nivel o curso actual del alumno y autoatribución de comportamientos socialmente responsables e intenciones a la base

La relación nivel-curso y autoatribución no muestra diferencia significativa en frecuencia. No así en la Escala de Intención mostrando una marcada diferencia, al alcanzar en la prueba T 0.0001, evidenciando que, a más años de permanencia en la universidad, aumenta la Intención de desarrollar comportamientos socialmente responsables, a diferencia de los alumnos recién ingresados. 
Tabla Nº7: T- Student de Frecuencia e Intención de autoatribución de comportamiento socialmente responsable de los alumnos de la Facultad de CS. Sociales de la UDEC; por Años de Permanencia; 2005.

\begin{tabular}{|c|c|c|c|c|c|c|c|c|c|}
\hline \multirow{2}{*}{\multicolumn{2}{|c|}{$\begin{array}{l}\text { AÑO- NIVEL } \\
\text { CURSO }\end{array}$}} & \multirow{2}{*}{$\mathrm{N}$} & \multirow{2}{*}{$\%$} & \multicolumn{6}{|c|}{ INTENCIÓN } \\
\hline & & & & Media & Std dev & $\operatorname{Pr}>F$ & Var & $\mathrm{T}$ & $\operatorname{Pr}>\mid t$ \\
\hline 1. & 1 año. & 157 & 61 & 84.81 & 16.2 & 0.0026 & VS & -3.99 & 0.0001 \\
\hline 2. & 4 año & 102 & 39 & 91.87 & 12.23 & & & & \\
\hline
\end{tabular}

\section{Relaciones no significativas}

Las variables que no muestran relación significativa de autoatribución de comportamientos socialmente responsables e intenciones a la base son: tipo de enseñanza media de procedencia lugar de residencia previo al ingreso a la universidad "ciudad de procedencia y apoyo económico recibido de la universidad (Factores Ambientales), como también la variable Rendimiento Académico (Factor Cognitivo).

\section{Conclusiones}

El estudio realizado permite sostener que los estudiantes de la facultad de ciencias sociales se autoatribuyen Comportamientos Socialmente Responsables, destacando mayor frecuencia en los comportamientos relacionados con el "respeto por los espacios compartidos", "autocuidado", "convivencia social" y "responsabilidad académica". Sin embargo, dado que reportan mayoritariamente no tener clara la intención a la base de estos comportamientos, no se puede concluir si son realmente comportamientos socialmente responsables. Un ejemplo de ello es que, en el ámbito académico, la intención autoatribuida es mayoritariamente relacionada con un interés de beneficio individual y no el beneficio común, por lo que "no" se le podría clasificar como comportamiento socialmente responsable.

Los estudiantes de sociología, en comparación con las otras 4 carreras, se autoatribuyen significativamente menos comportamientos socialmente responsables y menos intenciones de beneficio común, al alcanzar diferencia muy significativa en relación con las otras carreras (Factor Cognitivo). Si se considera que la autoatribución de un comportamiento es uno de los factores que orienta al individuo a actuar, la más baja autoatribución de comportamientos socialmente responsables en estudiantes de sociología, podría estar indicando 
que no se estaría logrando una de las competencias genéricas comprometidas en la formación y que sería necesario revisar el plan de formación de dicha carrera. Por una parte, los alumnos ingresan a la carrera con menor autoatribución de comportamientos socialmente responsables y menores intenciones de beneficio común, como también, egresarían con niveles más bajos en ambas variables, al ser comparados con sus compañeros de Facultad.

Con relación a la influencia de factores cognitivos en la autoatribución de comportamiento socialmente responsable, no hay relación entre ésta y el rendimiento académico, ni el nivel o curso del alumno $\left(1^{\circ} \circ 4^{\circ}\right)$. Sin embargo, se aprecian diferencias significativas en las intenciones a la base; de los alumnos de cuarto año, que muestran más intenciones de beneficio común, lo que podría estar reflejando un efecto favorable del contexto universitario, los planes de estudio, las oportunidades de experiencias socialmente responsables y de relaciones interpersonales que contribuyen al desarrollo o fortalecimiento de la RS.

En lo referente a factores biológicos que podrían influir en la responsabilidad social, las diferencias significativas encontradas de las mujeres por sobre los hombres en frecuencia de auto-atribución de comportamiento socialmente responsable comprueban, al menos en parte, el planteamiento de Barudy (2005) en cuanto a que las mujeres tienden más a desarrollar y mantener relaciones de ayuda. Sin embargo, este estudio no permite concluir si ello se puede atribuir a predisposición biológica, como lo plantea el autor, o a diferencias de género.

Finalmente, respecto de los factores ambientales, la característica que se relaciona de manera significativa con la auto-atribución en frecuencia e intención es el ejercicio de actividades de voluntariado de los alumnos y la pertenencia de ellos a familias que desarrollan dichas actividades. 


\section{Bibliografía}

Barudy Jorge; Dantagnan Maryorie (2005).

Los buenos tratos a la infancia-parentalidad, apego y resiliencia. GEDISA S.A. Barcelona.

Brunner, José Joaquín y Elacqua Gregory (2003).

Informe capital humano en Chile. Universidad Adolfo Ibáñez. Santiago de Chile.

Corporación Participa (2003).

La universidad construye país: La responsabilidad social de la universidad de cara al Chile del 2010. Corporación Participa. Santiago de Cile.

(2003).

Educando para la responsabilidad social. Universidad construye país: La Universidad en su función docente. Ediciones Proyecto Universidad Construye País. Santiago de Chile.

Fundación Prohumana (2002).

La responsabilidad social construyendo sentidos éticos para el desarrollo. Prohumana Ediciones. Santiago de Chile.

Navarro Saldaña, Gracia (2003-A).

“¿Qué entendemos por educación para la responsabilidad social?" En: Educando para la responsabilidad social. Universidad construye país: La universidad en su función docente. Edición Proyecto Universidad: Construye País. Santiago de Chile.

(2006).

"Comportamiento Socialmente Responsable". En: Responsabilidad social universitaria: Una manera de ser universidad, teoría y práctica en la experiencia chilena. Edición proyecto universidad construye país. Santiago de Chile.

Universidad Construye País (2001).

Seminario Asumiendo el país: Responsabilidad Social Universitaria. Ediciones Proyecto Universidad Construye País. Santiago de Chile. 


\section{Recursos electrónicos:}

Navarro Saldaña, Gracia (2003-B)

Universidad Construye País: Responsabilidad Social Universitaria. Una experiencia de Cooperación entre las Universidades Chilenas. Universidad de Concepción Chile. En:www.construyepais.cl/documentos/responsabilidad\%20social\%20extenso\%20 Nov\%202002.doc

Sánchez Henríquez, José (2007).

Política de Docencia de Pregrado. Dirección de Docencia Universidad de Concepción. En: http://www2.udec. $\mathrm{cl} / \sim$ dirdoc/perfeccionamiento/seminarios.php.

Universidad de Concepción (2006).

Seminario Internacional Comunicación Estratégica y Responsabilidad Social Universitaria Universidad de Concepción Chile. En:www2.udec.cl/seminario/ descargar.php?id=seminario. .pdf 